SPIN

Vol. 5, No. 3 (2015) 1550004 (10 pages)

(C) The Authors

DOI: $10.1142 / \mathrm{S} 2010324715500046$

\title{
Ultrafast and Distinct Spin Dynamics in Magnetic Alloys
}

\author{
I. Radu ${ }^{*, \dagger, * *, \dagger}$, C. Stamm ${ }^{\dagger, \dagger}$, A. Eschenlohr ${ }^{\dagger, \S \S}$, F. Radu*, R. Abrudan ${ }^{\S}$, \\ K. Vahaplar*, T. Kachel ${ }^{\dagger}$, N. Pontius ${ }^{\dagger}$, R. Mitzner ${ }^{\dagger}$, K. Holldack ${ }^{\dagger}$, A. Föhlisch ${ }^{\dagger}$, \\ T. A. Ostler", J. H. Mentink*, R. F. L. Evans", R. W. Chantrell", \\ A. Tsukamoto", A. Itoh", A. Kirilyuk*, A. V. Kimel* and Th. Rasing* \\ *Radboud University, Institute for Molecules and Materials \\ Heyendaalseweg 135, 6525 AJ Nijmegen, The Netherlands \\ $\dagger$ Institut für Methoden und Instrumentierung \\ der Forschung mit Synchrotronstrahlung \\ Helmholtz-Zentrum Berlin für Materialien und Energie GmbH \\ Albert-Einstein-Straße 15, 12489 Berlin, Germany \\ *nstitut für Komplexe Magnetische Materialien \\ Helmholtz-Zentrum Berlin für Materialien und Energie $\mathrm{GmbH}$ \\ Albert-Einstein-Straße 15, 12489 Berlin, Germany \\ ${ }^{\S}$ Institut für Experimentalphysik/Festkörperphysik \\ Ruhr-Universität Bochum, 44780 Bochum, Germany \\ IDepartment of Physics, University of York \\ Heslington, York YO10 5DD, United Kingdom \\ ${ }^{\|}$College of Science and Technology \\ Nihon University, 7-24-1 Funabashi, Chiba, Japan \\ **radu@tu-berlin.de
}

Received 23 May 2015

Accepted 26 May 2015

Published 20 August 2015

Controlling magnetic order on ultrashort timescales is crucial for engineering the next-generation magnetic devices that combine ultrafast data processing with ultrahigh-density data storage. An appealing scenario in this context is the use of femtosecond (fs) laser pulses as an ultrafast, external stimulus to fully set the orientation and the magnetization magnitude of a spin ensemble.

\footnotetext{
${ }^{* *}$ Corresponding author.

† Currently with the Institute for Optics and Atomic Physics Technical University Berlin, Germany.

\#Currently with the Department of Material Science, ETH Zürich Schafmattstr. 30, 8093 Zürich, Switzerland.

${ }^{\S}$ Currently with Fakultät für Physik, Universität Duisburg-Essen Lotharstr. 1, 47057 Duisburg, Germany.

This is an open access article published by World Scientific Publishing and distributed under the terms of the Creative Commons Attribution (CC BY) 4.0 License, which permits use, distribution and reproduction in any medium, provided the original author(s) and source are credited.
} 
Achieving such control on ultrashort timescales, e.g., comparable to the excitation event itself, remains however a challenge due to the lack of understanding the dynamical behavior of the key parameters governing magnetism: The elemental magnetic moments and the exchange interaction. Here, we investigate the fs laser-induced spin dynamics in a variety of multi-component alloys and reveal a dissimilar dynamics of the constituent magnetic moments on ultrashort timescales. Moreover, we show that such distinct dynamics is a general phenomenon that can be exploited to engineer new magnetic media with tailor-made, optimized dynamic properties. Using phenomenological considerations, atomistic modeling and time-resolved X-ray magnetic circular dichroism (XMCD), we demonstrate demagnetization of the constituent sub-lattices on significantly different timescales that depend on their magnetic moments and the sign of the exchange interaction. These results can be used as a "recipe" for manipulation and control of magnetization dynamics in a large class of magnetic materials.

Keywords: Ultrafast spin dynamics; element-specific magnetization; atomistic spin simulations.

\section{Introduction}

Femtosecond (fs) laser-induced controlling of ferromagnetic order ${ }^{1}$ has intrigued researchers since the pioneering work of Beaurepaire et al. ${ }^{2}$ who found that fs optical excitation of ferromagnetic $\mathrm{Ni}$ can demagnetize the sample on a sub-picosecond time-scale. This demagnetization event was much faster than one might expect supposing that the demagnetization process is defined by the characteristic time of the spin-lattice relaxation in the ground state. Later, it was demonstrated that circularly polarized laser pulses could act as equally short and relatively strong effective magnetic field pulses, ${ }^{3}$ even leading to fs laser-induced switching of the magnetization. ${ }^{4}$ These intriguing observations have triggered intense experimental and theoretical efforts to understand the fundamental processes underpinning such ultrafast laser-induced dynamics. ${ }^{1}$ Although a few models of laser-induced demagnetization of simple ferromagnets such as $\mathrm{Ni}$, $\mathrm{Fe}, \mathrm{Co}$ and $\mathrm{Gd}$ have been suggested ${ }^{5-7}$ the physics of ultrafast magnetic phenomena in systems with two or more magnetic sublattices ${ }^{8-13}$ still challenges our understanding. Yet many magnetization dynamics studies were performed with magnetic compounds containing at least two magnetic elements, like the well-known Permalloy, ${ }^{14-16}$ orthoferrites, ${ }^{3}$ manganites ${ }^{17}$ and yttrium-iron-garnets. ${ }^{18}$ In the conventional macrospin approximation, ${ }^{1}$ the constituent magnetic sublattices of an alloy are represented by a single macrospin [see Fig. 1(a)], which is used to describe their static and dynamic properties. This implies that two exchange-coupled magnetic sublattices are dynamically indistinguishable and thus would lose their magnetization on the very same time-scale upon ultrafast (fs) laser excitation. However, recent experiments using fs X-ray magnetic circular dichroism (XMCD) have revealed distinctly different magnetization dynamics of the constituent magnetic moments in a $\mathrm{Gd}(\mathrm{FeCo})$ alloy excited by a fs optical pulse. ${ }^{10}$ Moreover, it was subsequently shown ${ }^{19}$ that these distinct dynamics in combination with the exchange interaction between the sublattices, can lead to a deterministic switching of this ferrimagnetic $\mathrm{Gd}(\mathrm{FeCo})$ by an ultrafast heat pulse alone. ${ }^{20}$ These results lead to the intriguing question of whether such element-specific spin dynamics is a general phenomenon or something strictly related

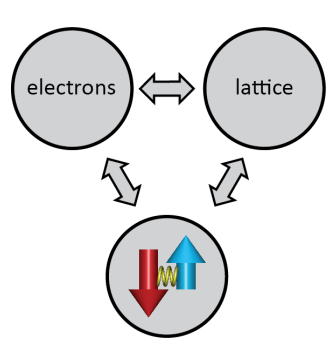

(a)

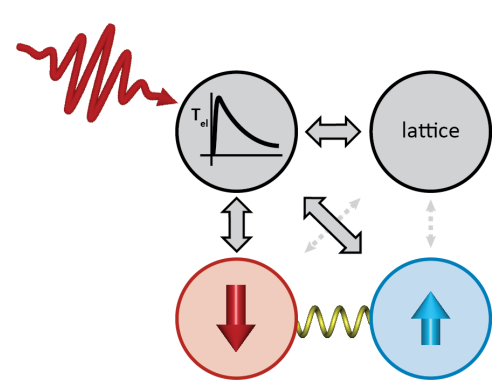

(b)
Fig. 1. Schematic illustration of the conventional (a) and of the ultrafast and distinct (b) spin dynamics. In the conventional picture (a) the dynamics of spin systems consisting of several magnetic elements is treated in the macrospin approximation where all the constituent elements are treated as a single macrospin. Within the ultrafast and distinct spin dynamics picture (b), one accounts for the dynamics of each magnetic constituent of a multi-component magnetic alloy separately, their dynamics being governed by the magnitude of their magnetic moment (the arrows in the figure) and by their mutual exchange interaction (the springs in the figure). 
to a narrow class of GdFe-like ferrimagnetic materials?

Here, we investigate the dynamics of multi-sublattice magnetic materials with both ferromagnetic and antiferromagnetic coupling between their sublattices. Using phenomenological modeling and atomistic spin simulations, we show that their demagnetization dynamics is distinct and elementspecific. These simulations are fully supported by element-specific fs time-resolved XMCD experiments on various $\mathrm{NiFe}$ and rare earth-transition metal (RE-TM) alloys, which provide evidence for a demagnetization time that scales with the elemental magnetic moment and varies with the sign of the exchange interaction. As such, one can tune the speed of magnetization processes in multi-sublattice alloys by properly choosing the magnitude of the constituent magnetic moments and the sign of the exchange interaction that couples them. These results may lead to new design principles of magnetic media for fast and energy-efficient magnetic data manipulation and storage. ${ }^{12,13,23}$

\section{Phenomenological Model of Ultrafast Magnetization Dynamics in Ferromagnetic Alloys}

To gain a qualitative understanding of the ultrafast demagnetization dynamics in ferromagnetically ordered two-sublattice magnets, we employ a macroscopic theory developed originally by Baryakhtar ${ }^{21}$ for the description of the dynamics and relaxation of the macroscopic (sublattice) magnetization of ferromagnets and antiferromagnets. It was recently shown that this approach can qualitatively describe the spatially averaged and dominantly longitudinal dynamics obtained from atomistic spin dynamics simulations. ${ }^{8}$ Clearly, the Baryakhtar theory is not restricted to longitudinal dynamics, and it has recently been demonstrated ${ }^{22}$ for antiferromagnetic coupling in the exchange approximation, that in addition to the purely longitudinal solutions, the theory exhibits a second class of solutions, which for specific conditions allows for the appearance of transverse time evolution with the same timescale as the longitudinal exchange relaxation. For the present work, we are interested in a different regime, where both the relativistic relaxation as well as the exchange relaxation play an important role (regime 3 according to Ref. 8), and we will restrict ourselves to the class of longitudinal solutions only. This approach is supported by atomistic simulations, ${ }^{23}$ which show that even when the system exhibits a small canting before laser excitation, the fast initial relaxation directly after excitation, i.e., the ultrafast demagnetization, is dominantly longitudinal. Furthermore, all experiments that operate at macroscopic length scales, including the X-ray experiments we describe below, demonstrate a longitudinal evolution of the (sublattice) magnetization on the sub-picosecond timescale. Hence on macroscopic length scales, the longitudinal time-evolution seems to be the relevant one. Under these conditions, the equations of motion are:

$$
\begin{aligned}
& \dot{S}_{1}=\zeta_{1} H_{1}+\zeta_{e}\left(H_{1}-H_{2}\right), \\
& \dot{S}_{2}=\zeta_{2} H_{2}+\zeta_{e}\left(H_{2}-H_{1}\right),
\end{aligned}
$$

where $S_{i}$ denotes the macroscopic spin angular momentum of sublattice $i=1,2$, and $H_{i}=-\delta W / \delta S_{i}$ is the effective magnetic field for sublattice $i$, which is derived from the magnetic (Landau) free energy $W$. $\zeta_{i}$ are the relativistic relaxation parameters describing the transfer of angular momentum with the environment, while $\zeta_{e}$ describes the exchange relaxation responsible for transfer of angular momentum between the sublattices. Previous work ${ }^{8}$ considered both $\zeta_{i, e}$ and the free energy $W$ as phenomenological parameters. As a next step, to assess the influence of the concentration, we derive the macroscopic free energy from a microscopic Heisenberg model, which is also the basis for atomistic spin dynamics simulations. Hence, similar to the latter, we explicitly assume that the longitudinal time-evolution on the macroscopic scale originates from spatial averaging over the transverse dynamics of the atomistic spins. Using the methods described in e.g., Refs. 24-26, we obtain in the mean field approximation for classical spins and for small $S_{i}$ values:

$$
\begin{gathered}
W=\frac{1}{2 N_{1} \chi_{1}} S_{1}^{2}+\frac{1}{2 N_{2} \chi_{2}} S_{2}^{2}-\frac{2 N_{p}}{N_{1} N_{2}} J_{12} S_{1} S_{2} \\
\chi_{i}^{-1}=\frac{3}{\mu_{1}^{2}} k_{\mathrm{B}} T-2 z_{i i} J_{i i} .
\end{gathered}
$$

For a binary alloy, we can use $N_{1}=(1-x) N$, $N_{2}=x N, \quad N_{p}=x(1-x) z N, z_{11}=z_{21}=(1-x) z$, $z_{22}=z_{12}=x z$, where $x$ is the concentration of sublattice $2, z$ the average number of nearest neighbors 
in the alloy, and $N$ the total number of spins per unit volume. In addition, $\mu_{i}$ indicates the magnitude of the spin angular momentum in sublattice $i$ and $J_{i j}$ are exchange parameters between spins in sublattice $i$ and $j$ that we take for nearest neighbors only. Further, $k_{\mathrm{B}} T$ is the energy of the temperature fluctuations experienced by the spins, which we assume to be solely determined by the electron temperature.

In principle, it is possible to extract the relaxation parameters from microscopic theory in a similar way as first-principle calculations of the transverse Gilbert relaxation parameter. ${ }^{27,28}$ This will generally yield parameters that depend on the temperature, the exchange interactions and the concentration. Below, we will consider the relaxation parameters $\zeta_{i, e}$ as purely phenomenological, in order to gain qualitative understanding in the limits of small and large concentration.

To understand qualitatively how the demagnetization time changes by going from the pure element to the alloy, we consider first the limiting case of small $x$. In this limit, we simplify the theory using $\zeta_{e}(x) \ll \zeta_{i}$ for $x \ll 1$ and the transfer of angular momentum between the sublattices will in general be a small effect. Furthermore, this transfer is generally less effective for ferromagnetic coupling than for antiferromagnetic coupling, since the terms in $H_{1}-H_{2}$ partly cancel each other. Therefore, we can write the demagnetization rate after a (fs laserinduced) step-like change of the electron temperature as $\dot{S}_{1}=\zeta_{1} H_{1}$. Using Eqs. (2a) and (2b) with $\left.S_{1}\right|_{t=0}=N_{1} \mu_{1}(x)$ we find:

$$
\dot{S}_{1}(x)=\zeta_{1}(x)\left[3 k_{\mathrm{B}} T-(1-x) z J_{11}-x z J_{12}\right] / \mu_{1}(x) .
$$

As in the pure material, we find a demagnetization time that depends on the magnetic moment, and hence the $\mathrm{Ni}$ and $\mathrm{Fe}$ sublattices generally have distinct dynamics after laser excitation due to the difference in their magnetic moments. In addition, the concentration determines the sublattice magnetic moments and exchange coupling strength, which can be different from the pure material. This in turn leads to a change in demagnetization times compared to the pure material. Applying this to the $\mathrm{NiFe}$ alloy at small $\mathrm{Fe}$ concentration, we have $J_{\mathrm{NiNi}}<J_{\mathrm{NiFe}} \cdot{ }^{29}$ Since also $\mu_{\mathrm{Ni}}(0)<\mu_{\mathrm{Ni}}(x)^{30}$ (see also Supplementary Sec. 6), we anticipate the demagnetization time of $\mathrm{Ni}$ increases upon moderate doping with Fe.
Next, we investigate the limit of large concentration $(x \approx 0.5)$. As before, the relativistic contributions generally yield distinct dynamics, however the transfer of angular momentum between the sublattices may not be negligible anymore. For example, at high-temperature $\left(k_{\mathrm{B}} T \gg J_{i j}\right)$ we have $H_{1}-H_{2} \approx-3 k_{\mathrm{B}} T\left(\mu_{1}^{-1}-\mu_{2}^{-1}\right)=+3 k_{\mathrm{B}} T\left(\mu_{1}-\mu_{2}\right) /$ $\mu_{1} \mu_{2}$. The sign of this term depends on the difference $\mu_{1}-\mu_{2}$, which suggests an additional acceleration (deceleration) of sublattice 1 (2) when $\mu_{1}<\mu_{2}$. Unfortunately, it is not straightforward to apply this analysis to the case of $\mathrm{NiFe}$, since for large concentration the system is in the Invar region. In this regime, the $\mathrm{Ni}$ sublattice remains almost collinear with the average magnetization, but the $\mathrm{Fe}$ sublattice is essentially frustrated and noncollinear even at very low temperature. This frustration is caused by long-range oscillating exchange interactions $^{31}$ and due to local environment effects that yield a huge dispersion of the nearest-neighbor exchange parameters, ${ }^{32}$ which results in a decrease of the Curie temperature despite the fact that the magnitude of the exchange interactions increases. ${ }^{33}$ Although the assessment of such frustrated magnetism goes beyond the nearest-neighbor Heisenberg model employed here, we anticipate that an essentially noncollinear Fe environment can allow for an efficient transfer of angular momentum between the sublattices (large parameter $\zeta_{e}$ ), which in the high-temperature limit yields an acceleration of the demagnetization of $\mathrm{Ni}$, since $\mu_{\mathrm{Ni}}<\mu_{\mathrm{Fe}}$. This acceleration counteracts the deceleration due to the increase of $\mu_{\mathrm{Ni}}$, which in the Invar regime is almost twice as large as in pure Ni. Hence, the exchange relaxation in the Invar regime can make the dynamics of $\mathrm{Ni}$ and $\mathrm{Fe}$ more distinct than anticipated solely from the difference of the magnetic moments, which is indeed the trend observed in the atomistic and experimental results shown below.

\section{Atomistic Spin Simulations of Ultrafast Demagnetization}

Following the model predictions above, we have performed atomistic simulations of the response of $10^{6}$ ferromagnetically exchange-coupled $\mathrm{NiFe}$ as well as antiferromagnetically coupled GdFe spins to an ultrashort $60 \mathrm{fs}$ heat pulse, as explained in the Supplementary Information. The dynamics of each magnetic sublattice were obtained by solving a 
system of coupled stochastic Landau-LifshitzGilbert equations for individual atomic moments, which describe the transient dynamics of the system upon excitation with a fs laser pulse. ${ }^{34}$ For multi-component magnetic alloys one derives ${ }^{34,35}$ a characteristic longitudinal relaxation time ${ }^{\mathrm{a}}$ (i.e., demagnetization time) for each constituent component which scales with:

$$
\tau_{i} \approx \frac{\mu_{i}}{2 \lambda_{i} \gamma_{i} k_{\mathrm{B}} T},
$$

where $\mu_{i}$ is the moment associated with site $i, \lambda_{i}$ is the coupling constant to the thermal bath, $\gamma_{i}$ is the gyromagnetic ratio at site $i, k_{\mathrm{B}}$ is Boltzmann's constant and $T$ is the temperature of the bath. Consequently, if an alloy consists of two sublattices and $\mu_{1 \neq} \mu_{2}$, a rapid heating of the system to high temperatures will lead to different demagnetization dynamics of the two sublattices, despite the strong exchange interaction that couples them. The effect of $\lambda_{i}$ and $\gamma_{i}$ on the demagnetization time is discussed in Supplementary Sec. 7.

We have simulated both ferromagnetically coupled NiFe alloys as well antiferromagnetically coupled $\mathrm{GdFe}$ (ferrimagnetic) systems. Following Ref. 34, we coupled the spin system to the electron temperature that is calculated using the twotemperature model,${ }^{36}$ which describes the transient variation of the electron and phonon temperatures upon femtosecond laser excitation. The strength of the thermal fluctuations then scales with this timevarying temperature. For simplicity, the model assumes the same coupling constants for the different sublattices $\left(\lambda_{i}=0.01\right)$, and the magnetic moments were derived from XMCD measurements (see Supplementary Information). The NiFe exchange interactions were determined from the measured Curie temperature of the $\mathrm{NiFe}$ alloy samples as reported in Ref. 37. For instance, in the case of $\mathrm{Ni}_{50} \mathrm{Fe}_{50}$, this yields a total exchange per spin of $260 \mathrm{meV}$. The results of the $\mathrm{Ni}_{50} \mathrm{Fe}_{50}$ simulations are displayed in Fig. 2(a), showing clearly a faster demagnetization for Ni than for Fe. This conclusion holds for the full stoichiometry range of the NiFe alloys, as detailed in the Supplementary Sec. 6 .

To simulate the antiferromagnetically coupled $\mathrm{GdFe}$ system, we used the exchange constants obtained by fitting the temperature-dependent

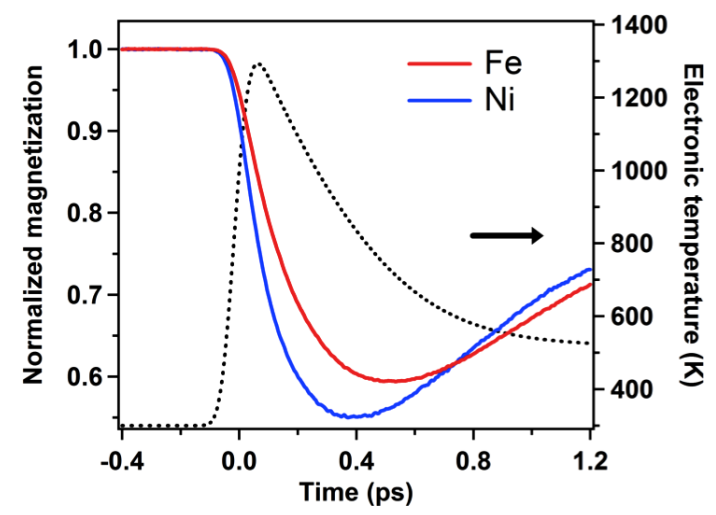

(a)

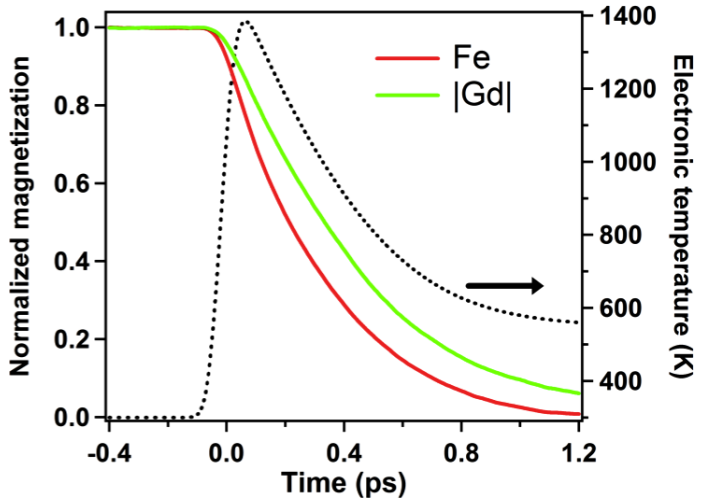

(b)

Fig. 2. Computed transient dynamics of the constituent magnetic moments in FeNi and GdFe alloys using the atomistic spin model. The response of the longitudinal component of the magnetization of $\mathrm{Ni}$ and $\mathrm{Fe}$ in $\mathrm{Ni}_{50} \mathrm{Fe}_{50}$ alloy (a) and of $\mathrm{Fe}$ and $\mathrm{Gd}$ in $\mathrm{Gd}_{24} \mathrm{Fe}_{76}$ alloy (b) to a 60 fs laser pulse. The dashed lines in both panels display the transient electronic temperature used in the simulation. The starting temperature for the simulation is $300 \mathrm{~K}$. Note that in panel (b) the Fe and Gd curves are merging together around $1.8 \mathrm{ps}$ (see Supplementary Information Sec. 3).

XMCD data measured for the Fe and Gd sublattices. ${ }^{35}$ Taking into account the number and type of neighbors, the total effective $\mathrm{Fe}-\mathrm{Gd}$ exchange is $30 \mathrm{meV}$. The results of the $\mathrm{GdFe}$ simulations are displayed in Fig. 2(b), showing a dissimilar demagnetization dynamics of $\mathrm{Fe}$ and $\mathrm{Gd}$ with $\mathrm{Fe}$ being much faster than Gd.

Hence, distinct timescales for the demagnetization of magnetic sublattices have been computed for both ferromagnetic and antiferromagnetic coupling between the sublattices. Thus, this appears to be a general phenomenon for multi-sublattice magnets. 


\section{Ultrafast Dynamics of the Elemental Magnetic Moments from Time-Resolved XMCD Experiments}

To compare these theoretical predictions and simulations with experiments, we measured the transient demagnetization dynamics of the magnetic sublattices in $\mathrm{NiFe}$ and GdFe alloys ${ }^{\mathrm{b}}$ as well as their respective pure elements, employing the element-specific XMCD technique in transmission at the femto-slicing facility ${ }^{38,39}$ of the HelmholtzZentrum Berlin. In addition, we have also investigated a ferrimagnetic $\mathrm{DyCo}_{5}$ (i.e., $\mathrm{Dy}_{16.7} \mathrm{Co}_{83.3}$ ) alloy ${ }^{40}$ that shows the largest difference between the magnitudes of the constituent magnetic moments (see Supplementary Fig. 3). The experiments were performed in a stroboscopic pump-and-probe mode, where the metallic samples were excited with $60 \mathrm{fs}$ laser pulses of $1.55 \mathrm{eV}$ photon energy. Subsequent laser-induced magnetization dynamics were probed by measuring the XMCD changes in transmission with circularly polarized 100 fs X-ray pulses ${ }^{39}$ tuned at the $\mathrm{L}_{3}$ edges of $\mathrm{Ni}(853 \mathrm{eV}), \mathrm{Fe}(707 \mathrm{eV})$, Co $(778 \mathrm{eV})$ and at the $\mathrm{M}_{5}$ edges of $\mathrm{Gd}(1189 \mathrm{eV})$ and Dy $(1292 \mathrm{eV})$. At these energies, we probe the dynamics of the $3 d$ magnetic moment of $\mathrm{Ni}, \mathrm{Fe}$, Co and the $4 f$ moment of $\mathrm{Gd}$ and Dy, respectively. We point out that at these absorption edges, there is a nonambiguous relationship between the measured XMCD signal and the elemental magnetic moments ${ }^{41}$ that remains valid even in the high nonequilibrium regime after laser excitation. ${ }^{42}$ The XMCD measurements were performed on $\mathrm{Fe}, \mathrm{Ni}$, $\mathrm{Ni}_{50} \mathrm{Fe}_{50}, \mathrm{Ni}_{80} \mathrm{Fe}_{20}, \mathrm{Dy}_{16.7} \mathrm{Co}_{83.3}$ and $\mathrm{Gd}_{25} \mathrm{Fe}_{66} \mathrm{Co}_{9}$ samples at $300 \mathrm{~K}$ except for $\mathrm{DyCo}_{5}$, which were done at $100 \mathrm{~K}$ (under laser exposure the effective sample temperature of $\mathrm{DyCo}_{5}$ rose well above its compensation temperature of $120 \mathrm{~K}$ due to a permanent heating effect, as deduced from the polarity of the XMCD signal and the elemental hysteresis). For $\mathrm{Gd}(\mathrm{FeCo})$, the magnetization compensation temperature was $250 \mathrm{~K}$. The typical absorbed laser fluence was $\sim 6.8 \mathrm{~mJ} / \mathrm{cm}^{2}$ for $\mathrm{NiFe}$ alloys, $\sim 10.1 \mathrm{~mJ} / \mathrm{cm}^{2}$ for $\mathrm{DyCo}_{5}$ while for $\mathrm{Gd}(\mathrm{FeCo})$ it was $\sim 4.5 \mathrm{~mJ} / \mathrm{cm}^{2}$. The $\mathrm{Fe}, \mathrm{Ni}$ and $\mathrm{NiFe}$ films exhibit an in-plane magnetization orientation, while $\mathrm{Gd}(\mathrm{FeCo})$ and $\mathrm{DyCo}_{5}$ have an out-of-plane magnetic anisotropy. The samples are poly-crystalline except for $\mathrm{Gd}(\mathrm{FeCo})$ which is amorphous.

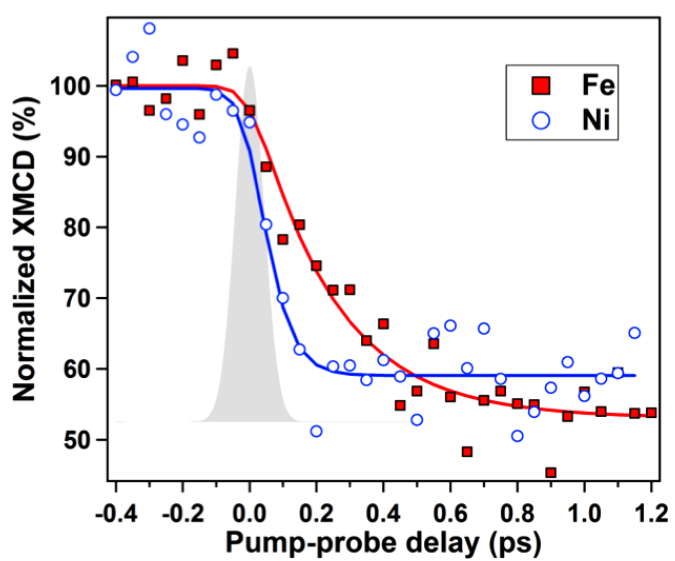

(a)

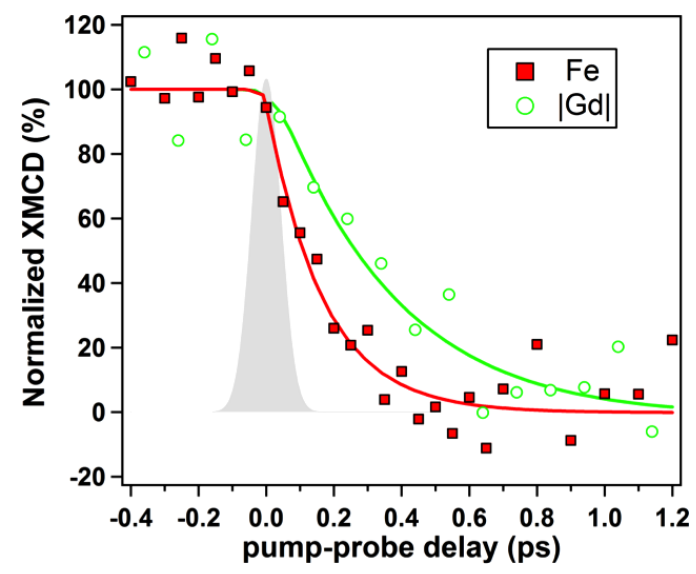

(b)

Fig. 3. Element-specific demagnetization dynamics of the constituent magnetic moments in $\mathrm{FeNi}$ and $\mathrm{GdFe}$ alloys measured by time-resolved XMCD with fs time-resolution. Element-specific demagnetization measured in (a) ferromagnetic $\mathrm{Ni}_{50} \mathrm{Fe}_{50}$ at the $\mathrm{Fe} \mathrm{L}_{3}$ and $\mathrm{Ni} \mathrm{L}_{3}$ edges and (b) in ferrimagnetic $\mathrm{Gd}_{25} \mathrm{Fe}_{66} \mathrm{Co}_{9}$ alloy at the $\mathrm{Fe} \mathrm{L}_{3}$ and $\mathrm{Gd} \mathrm{M}_{5}$ edges. The solid lines are fits according to a bi-exponential fit function (see Eq. (5)). The Gaussian profiles depict the time resolution of the XMCD measurements of $130 \mathrm{fs}$.

The results of the element-specific laser-induced demagnetization of ferromagnetic $\mathrm{Ni}_{50} \mathrm{Fe}_{50}$ are shown in Fig. 3(a). Upon fs laser excitation, the alloy demagnetizes to about $50 \%$ at both the Fe and $\mathrm{Ni}$ edges. Although a similar degree of demagnetization is achieved for both elements, their transient demagnetization behavior is considerably different: While Fe is demagnetized within $\sim 800 \mathrm{fs}$, it takes only $\sim 300 \mathrm{fs}$ for $\mathrm{Ni}$ to demagnetize to the same extent as $\mathrm{Fe}$, in qualitative agreement with the

bThe GdFe sample contained a small percentage (9\%) of Co to control the magnetic anisotropy - for more details, see Supplementary Information Sec. 1. 
predictions from Fig. 2(a). Although less pronounced, we measure similar distinct demagnetization dynamics of the $\mathrm{Fe}$ and $\mathrm{Ni}$ moments in a $\mathrm{Ni}_{80} \mathrm{Fe}_{20}$ sample with again $\mathrm{Fe}$ being slower than $\mathrm{Ni}$ (see Supplementary Fig. 7).

Essentially, a similar decoupled demagnetization behavior of the constituent, but antiferromagnetically coupled, magnetic moments is encountered for the ferrimagnetic $\mathrm{Gd}(\mathrm{FeCo})$ alloy, as shown in Fig. 3(b). In this case, the demagnetization of Fe takes $\sim 400 \mathrm{fs}$ while $\mathrm{Gd}$ demagnetizes within $\sim 1.2 \mathrm{ps}$. Due to the lower $T_{\mathrm{C}}$ of $\sim 550 \mathrm{~K}$ (see Ref. 35), here we totally quench the magnetic order at both $\mathrm{Fe}$ and Gd edges i.e., achieve a demagnetization degree of $100 \%$. This dynamic decoupling of $\mathrm{Fe}$ and $\mathrm{Gd}$ is however also encountered for much lower demagnetization degrees down to $\sim 40 \%$ (see Supplementary Fig. 8).

For the ferrimagnetic alloy $\mathrm{DyCo}_{5}$, according to Eq. (4) and given the different values of the constituent Co $\left(\sim 1.52 \mu_{\mathrm{B}}\right)$ and Dy $\left(\sim 8.91 \mu_{\mathrm{B}}\right)$ magnetic moments (see Supplementary Fig. 3), we expect a large difference between their dynamics. Indeed, as shown in Supplementary Fig. 9, Co demagnetizes down to $\sim 50 \%$ within $\sim 350$ fs while it takes several ps for Dy to reach the same demagnetization level.

To extract the time constants of the measured demagnetization transients, we have used the following bi-exponential fit function:

$$
\begin{aligned}
f(t)= & g(t) \\
& \otimes\left[A-B \cdot \exp \left(-\frac{t}{\tau_{D}}\right)+C \cdot \exp \left(-\frac{t}{\tau_{R}}\right)\right],
\end{aligned}
$$

where $\tau_{D}$ and $\tau_{R}$ are the demagnetization and relaxation time constants, $g(t)$ is the Gaussian function describing the time resolution of the experiment, $B$ and $C$ are the exponential amplitudes and $A$ is the value of the transient signal at negative delays. The demagnetization time constants obtained from the fits are listed in Table 1 of Supplementary Information and plotted in Fig. 4(a). For comparison, we also show the demagnetization time constants of the elemental $\mathrm{Fe}, \mathrm{Ni}, \mathrm{Co}$ and $\mathrm{Gd}$ samples. The demagnetization of pure Co and Gd are taken from Refs. 5 and 43, respectively. For demagnetization of pure Ni, please see also Ref. 44 . We note that these demagnetization time constants are extracted from demagnetization transients (both

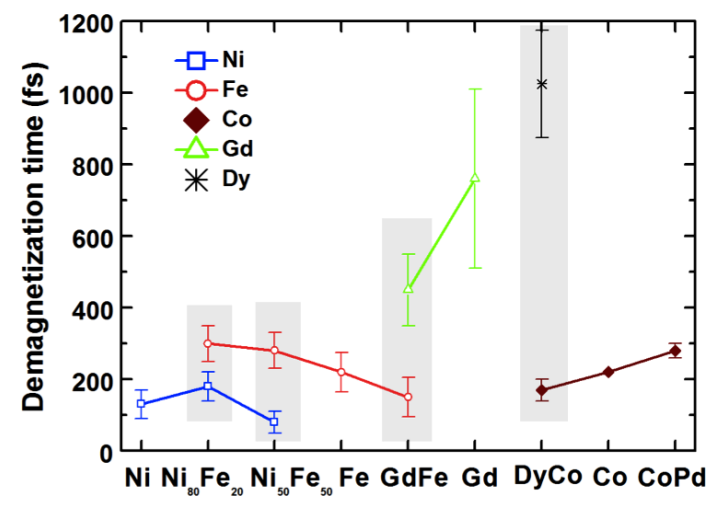

(a)

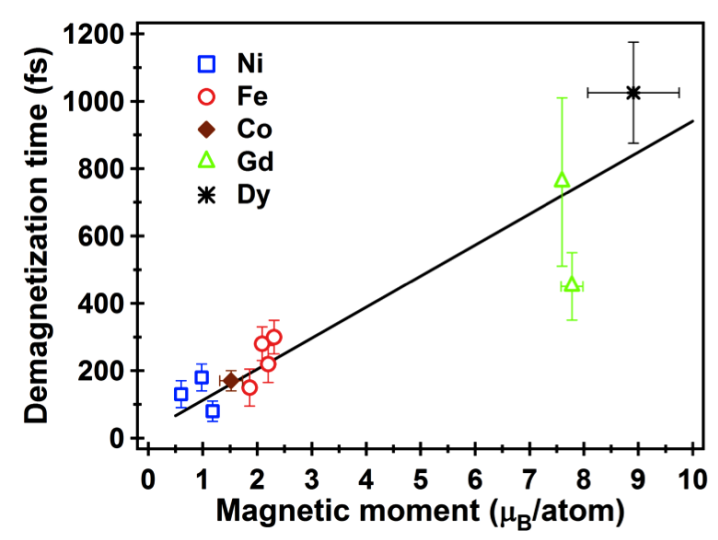

(b)

Fig. 4. The experimental demagnetization time constants measured for single $\mathrm{Ni}, \mathrm{Fe}, \mathrm{Co}$ and Gd elements and their alloys. In panel (a), we show the overview of the demagnetization time constants of the elemental magnetic moments in the $\mathrm{Ni}_{80} \mathrm{Fe}_{20}$, $\mathrm{Ni}_{50} \mathrm{Fe}_{50}, \mathrm{DyCo}_{5}$ and $\mathrm{Gd}_{25} \mathrm{Fe}_{66} \mathrm{Co}_{9}$ alloys and of the pure $\mathrm{Fe}, \mathrm{Ni}$, Co and Gd samples. The solid lines are guides to the eye. Demagnetization time constants of pure Co and Gd are taken from Refs. 5 and 43, respectively, while the demagnetization of Co in CoPd alloy is taken from Ref. 45 . The gray regions emphasize the dissimilar demagnetization time constants of the elements in the same alloy. Panel (b) shows the dependence of the demagnetization time constants from panel (a) on the magnitude of the elemental magnetic moments for pure elements and their alloys. The solid line is a linear fit to the data.

the alloys and their elemental counterparts) that exhibit similar demagnetization degrees $\Delta M / M_{0}$ of around $50 \%$, and thus we can treat and compare these demagnetization times on equal footing. In Fig. 4(b) we show the deduced demagnetization constants versus magnetic moment for all measured samples. One observes a clear scaling behavior between the demagnetization time and the magnitude of the magnetic moment. A quantitative estimate for the variation of the demagnetization time per magnetic moment unit is obtained by fitting the 


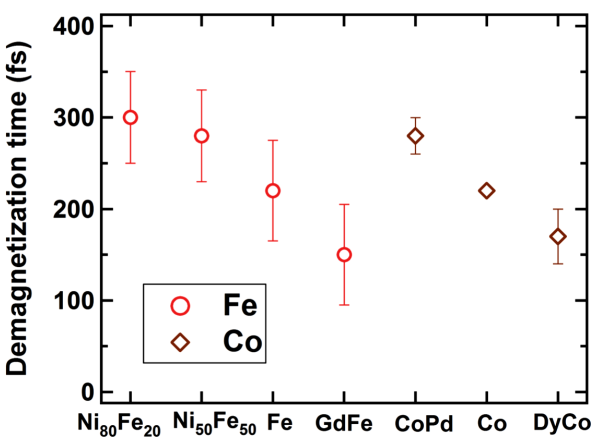

Fig. 5. The dependence of the demagnetization times for Fe and Co on the magnetic ordering type of the host alloys. Comparison of the demagnetization time constants measured for $\mathrm{Fe}$ and $\mathrm{Co}$ in ferromagnetic $\mathrm{FeNi}$ and $\mathrm{CoPd}$, ferrimagnetic GdFeCo and DyCo as well as for pure elements. The demagnetization time constants for pure Co and Co in CoPd are taken from Refs. 5 and 45, respectively.

data using a linear fit function [the solid line in Fig. 4 (b)], which gives us a value of $90 \pm 20 \mathrm{fs} / \mu_{\mathrm{B}}$.

Another important piece of information provided by these data is the dependence of the demagnetization time on the magnetic ordering type of the samples. This is shown in Fig. 5 where the demagnetization time constants of $\mathrm{Fe}$ and $\mathrm{Co}$ are plotted for pure samples, ferromagnetic FeNi and CoPd and ferrimagnetic (i.e., antiferromagnetic coupling) $\mathrm{Gd}$ (FeCo) and DyCo alloys. For a ferromagnetic type of coupling, both the Fe and Co moments demagnetize slower than the pure material while the opposite is observed in the antiferromagnetically coupled GdFe and DyCo. Please note that the very same behavior is encountered when normalizing the elemental demagnetization times to the corresponding magnetic moment magnitudes.

\section{Discussion}

There are three major effects that can be observed in the overview of the demagnetization time constants shown in Figs. 4 and 5: (i) The distinctly different demagnetization times of the elemental magnetic moments in the ferromagnetic $\mathrm{NiFe}$ and ferrimagnetic $\mathrm{Gd}(\mathrm{FeCo})$ and $\mathrm{DyCo}_{5}$ alloys [the highlighted regions in Fig. 4(a)], (ii) the demagnetization times scale approximately with the magnitude of the elemental magnetic moment [see Fig. 4(b)] for the pure samples of $\mathrm{Ni}, \mathrm{Fe}, \mathrm{Co}$ and $\mathrm{Gd}$ as well as for their alloys and (iii) the demagnetization time of a specific element (e.g., Fe or Co) changes with the exchange interaction type of the host alloy i.e., it is longer in ferromagnetic $\mathrm{FeNi}$ and shorter in antiferromagnetically coupled $\mathrm{Gd}(\mathrm{FeCo})$ and $\mathrm{DyCo}_{5}$ compared to their pure $\mathrm{Fe}$ and Co counterparts (see Fig. 5).

Both the XMCD data and the simulations show that, despite the strong ferromagnetic exchange coupling between the $\mathrm{Ni}$ and $\mathrm{Fe}$ sublattices in $\mathrm{NiFe}$, they apparently lose their net magnetization in a very dissimilar manner. The same situation is also observed in ferrimagnetic $\mathrm{Gd}(\mathrm{FeCo})$ and $\mathrm{DyCo}_{5}$, where two antiferromagnetically coupled $\mathrm{Gd}$ and $\mathrm{Fe}$ or Dy and Co sublattices demagnetize on substantially different time-scales. Thus, we observe the same transient decoupling of the exchange-coupled magnetic moments in two material systems that are strikingly different, i.e., ferromagnetic versus antiferromagnetic coupling, itinerant versus localized type of magnetic ordering and in-plane versus outof-plane magnetic anisotropy. Consequently this distinct dynamics seems to be a general property of multi-elemental magnetic compounds that are driven nonadiabatically to a highly nonequilibrium state by fs laser excitation. Moreover, in agreement with Eqs. (3) and (4), the observed characteristic demagnetization times scale with the magnetic moment of the sublattices i.e., the larger the magnetic moment the slower the demagnetization process [see Fig. 4(b)]. For the materials investigated here, we obtain a characteristic change of the demagnetization time per magnetic moment of $90 \pm 20 \mathrm{fs} / \mu_{\mathrm{B}}$. Consequently, although in NiFe, Fe demagnetizes slower than $\mathrm{Ni}$, in $\mathrm{Gd}(\mathrm{FeCo})$ the demagnetization of the Fe-sublattice is much faster than the demagnetization of Gd. This distinct dynamics is even more pronounced in $\mathrm{DyCo}_{5}$ given the large difference (a factor of 6 ) between the Dy and Co magnetic moments.

Furthermore, the antiferromagnetic coupling in $\mathrm{Gd}(\mathrm{FeCo})$ and $\mathrm{DyCo}_{5}$ yields faster demagnetization of both sublattices when compared to their pure elements. The ferromagnetic coupling in $\mathrm{Ni}_{50} \mathrm{Fe}_{50}$, on the other hand, yields faster demagnetization of $\mathrm{Ni}$ and slower demagnetization of $\mathrm{Fe}$, despite the fact that the magnetic moment of $\mathrm{Ni}$ increases (see Supplementary Fig. 3 for variation of the magnetic moment with the sample stoichiometry). In $\mathrm{Ni}_{80} \mathrm{Fe}_{20}$, the exchange coupling between the sublattices is not effective enough (see Table 2 in Supplementary Information) to compensate for the increased magnetic moment, yielding an increase of 
the demagnetization time of $\mathrm{Ni}$ compared to the pure case.

Overall, these results show that is possible to control the dynamics of the constituent sublattices in magnetic alloys by changing the magnitude of the magnetic moment of the constituent elements, the sign and strength of the exchange interaction or a combination thereof. Moreover, knowing the key parameters that govern the ultrafast magnetization dynamics in alloys, we can extend this "recipe" and hypothesize that magnetization dynamics can be engineered the very same way in artificial magnetic structures mimicking the intrinsic properties of alloys, as for instance in synthetic ferrimagnets. ${ }^{46}$

Before concluding the paper, we would like to discuss the relevance of the recent transversal magneto-optical Kerr effect (TMOKE) results by Mathias et al. ${ }^{15}$ in the context of our study. These authors used time-resolved magneto-optics in reflection to investigate the laser-induced demagnetization of pure $\mathrm{Fe}, \mathrm{Ni}$ and of a permalloy $\left(\mathrm{Ni}_{80} \mathrm{Fe}_{20}\right)$ sample. By tuning the probing VUV photon energy at the $\mathrm{M}_{3,2}(3 p-3 d$ transitions $)$ resonances of $\mathrm{Ni}$ $(\sim 67 \mathrm{eV})$ and $\mathrm{Fe}(\sim 54 \mathrm{eV})$ they report that $\mathrm{Fe}$ demagnetizes quicker than $\mathrm{Ni}$ in pure samples while in Permalloy both elements exhibit the same demagnetization time (about $240 \mathrm{fs}$ ) and a time lag in the onset of their respective transient dynamics. There are two major differences between our work and the study of Ref. 15: (i) Their measurements were performed in reflection geometry and (ii) magnetization dynamics has been probed at the $\mathrm{M}_{3,2}$ elemental edges in the VUV spectral range. Regarding point (i), magneto-optical X-ray measurements performed in reflection do not provide a direct, quantitative measure of the elemental magnetic moment, which is at variance compared to their transmission counterparts. ${ }^{41}$ This is because the magneto-optical signal measured in reflection consists of an admixture of dispersive and absorptive parts of the dielectric function, and as such the use of the sum rules ${ }^{c}$ to determine the magnetic moment is inapplicable. Second, in the VUV spectral range the energies corresponding to the spin-orbit and the exchange interactions are of comparable magnitude, which leads to a strong distortion of the $3 p$ electronic states of $\mathrm{Ni}$ and $\mathrm{Fe}$ (the $\mathrm{M}_{3}$ and $\mathrm{M}_{2}$ absorption edges are essentially overlapped) and consequently, one cannot apply the sum rules to obtain the magnitude of the magnetic moment (see e.g., Ref. 47). This is valid for both reflection and absorption measurements. Moreover, in the VUV range, the close energetic proximity of $\mathrm{Fe}$ and $\mathrm{Ni}$ absorption edges results in an overlap of their respective magnetic signals; this renders, for such alloys, the interpretation of the magnetization data in terms of pure elemental response more difficult and ambiguous. Also, the time-resolved TMOKE measured in the VUV range might be susceptible to magneto-optical artifacts, as has been recently pointed out by Vodungbo et $a .^{48}$ Thus, the TMOKE signal might contain nonmagnetic contributions that complicate the interpretation of the data as being only of magnetic origin.

In short, due to their measurement specificity, the results of Mathias et al. ${ }^{15}$ do not provide information about the dynamics of the elemental magnetic moment in their samples. This is at variance with our present work performed in the soft X-ray spectral range, which allows the accurate application of the magneto-optical sum rules and thus providing a nonambiguous relationship between the measured signal and the magnetic moment. As such, there is no common basis for comparing our results with those reported in Ref. 15 as this work is essentially measuring different observables.

\section{Conclusion}

To conclude, our atomistic spin modeling and phenomenological theory, supported by element-specific fs XMCD experiments, demonstrate the possibility of tuning the dynamics of multi-sublattice ferromagnets and ferrimagnets by their composition, which determines their magnetic moments and the strength of the exchange interaction. This can lead to distinctly different demagnetization times even for the same element in different alloys. In particular, we have shown that for antiferromagnetic coupling, exchange relaxation accelerates the demagnetization of both sublattices, while with ferromagnetic coupling, the dynamics of one sublattice is accelerated while the other is slowed down. Furthermore, we have shown that, by tuning the composition we can control the distinctiveness of the

cThe magneto-optical sum rules relate the absorption cross-section with the ground-state expectation values of the spin $\left\langle S_{z}\right\rangle$ and orbital $\left\langle L_{z}\right\rangle$ moments. 
sublattice dynamics. This allows designing new magnetic materials, for example using exchange coupled multilayers, which combine desirable properties for future recording media. ${ }^{12,13,23}$

\section{Acknowledgments}

Funding from the European Community's Seventh Framework Program (FP7/2007-2013) Grants No. NMP3-SL-2008-214469 (UltraMagnetron), No. 214810 (FANTOMAS) and No. 281043 (FEMTOSPIN) and ERC Grant No. 257280 (Femtomagnetism) as well as Grant No. 226716 and ERC-2013AdG339813-EXCHANGE, the German Federal Ministry of Education and Research (BMBF) Grant No. 05K10PG2 (FEMTOSPEX), the Foundation for Fundamental Research on Matter (FOM) and the Netherlands Organization for Scientific Research (NWO) is gratefully acknowledged.

\section{References}

1. A. Kirilyuk, A. V. Kimel and Th. Rasing, Rev. Mod. Phys. 82, 2731 (2010).

2. E. Beaurepaire, J.-C. Merle, A. Daunois and J.-Y. Bigot, Phys. Rev. Lett. 76, 4250 (1996).

3. A. V. Kimel et al., Nature (London) 429, 850 (2004).

4. C. D. Stanciu et al., Phys. Rev. Lett. 99, 047601 (2007).

5. B. Koopmans et al., Nature Mater. 9, 259 (2010).

6. M. Battiato, K. Carva and P. M. Oppeneer, Phys. Rev. Lett. 105, 027203 (2010).

7. J.-Y. Bigot, M. Vomir and E. Beaurepaire, Nature Phys. 5, 515 (2009).

8. J. Mentink et al., Phys. Rev. Lett. 108, 057202 (2012).

9. S. Wienholdt et al., Phys. Rev. B. 88, 020406 (2013).

10. I. Radu et al., Nature (London) 472, 205 (2011).

11. N. Bergeard et al., Nature Commun. 5, doi: 10.1038/ ncomms4466 (2014).

12. S. Mangin et al., Nature Mater. 13, 286 (2014).

13. C. H. Lambert et al., Science 345, 1337 (2014).

14. C. La-O-Vorakiat et al., Phys. Rev. Lett. 103, 257402 (2009).

15. S. Mathias et al., P. Natl Acad. Sci. USA 109, 4792 (2012).
16. I. Radu et al., Phys. Rev. Lett. 102, 117201 (2009).

17. T. Lottermoser et al., Nature 430, 541 (2004).

18. S. O. Demokritov et al., Nature 443, 430 (2006).

19. T. Ostler et al., Nature Commun. 3, 666 (2012).

20. J. Barker et al., Scientific Reports 3, 03262 (2013).

21. V. G. Baryakhtar Sov. Phys. JETP 60, 863 (1984).

22. V. G. Baryakhtar, V. I. Butrim and B. A. Ivanov, JETP Lett. 98, 289 (2013).

23. R. Evans et al., Appl. Phys. Lett. 104, 082410 (2014).

24. H. Falk, Am. J. Phys. 38, 858 (1970).

25. O. F. Abubrig et al., Physica A 296, 437 (2001).

26. J. W. Negele and H. Orland, Quantum ManyParticle Systems (Westview Press, Boulder USA, 1988).

27. H. Ebert et al., Phys. Rev. Lett. 107, 066603 (2011).

28. A. A. Starikov et al., Phys. Rev. Lett. 105, 236601 (2010).

29. P. Yu et al., Phys. Rev. B 77, 054431 (2008).

30. B. Glaubitz et al., J. Phys.: Condens. Matter 23, 254210 (2011).

31. M. van Schilfgaarde et al., Nature 400, 46 (1999).

32. A. V. Ruban et al., Phys. Rev. B 71, 054402 (2005).

33. J. Kudrnovský et al., Phys. Rev. B 77, 224422 (2008).

34. N. Kazantseva et al., Europhys. Lett. 81, 27004 (2008).

35. T. Ostler et al., Phys. Rev. B. 84, 024407 (2011).

36. S. I. Anisimov, B. L. Kapeliovich and T. L. Perelman, Sov. Phys. JETP 39, 375 (1974).

37. E. H. Wasserman, in Ferromagnetic Materials, Vol. 5 (Elsevier, Amsterdam, The Netherlands, 1990).

38. C. Stamm et al., Nature Mater. 6, 740 (2007).

39. S. Khan et al., Phys. Rev. Lett. 97, 074801 (2006).

40. F. Radu, R. Abrudan, I. Radu, D. Schmitz and H. Zabel, Nature Commun. 3, 715 (2012).

41. C. T. Chen et al., Phys. Rev. Lett. 75, 152 (1995).

42. K. Carva et al., Europhys. Lett. 86, 57002 (2009).

43. M. Wietstruk et al., Phys. Rev. Lett. 106, 127401 (2011).

44. C. Stamm et al., Phys. Rev. B 81, 104425 (2010).

45. C. Boeglin et al., Nature 465, 458 (2010).

46. R. Evans, T. Ostler, R. Chantrell, I. Radu and Th. Rasing, Appl. Phys. Lett. 104, 082410 (2014).

47. D. Zhao et al., J. Appl. Phys. 84, 2858 (1998).

48. B. Vodungbo et al., Phys. Rev. X 3, 038001 (2013). 